\title{
Inductive learning of the surgical workflow model through video annotations
}

\author{
Hirenkumar Nakawala, Elena De Momi, Laura Erica Pescatori, Anna Morelli, Giancarlo Ferrigno \\ Department of Electronics, Information and Bioengineering (DEIB) \\ Politecnico di Milano \\ Milan, Italy \\ \{hirenkumar.nakawala, elena.demomi, giancarlo.ferrigno\}@polimi.it
}

\begin{abstract}
Surgical workflow modeling is becoming increasingly useful to train surgical residents for complex surgical procedures. Rule-based surgical workflows have shown to be useful to create context-aware systems. However, manually constructing production rules is a time-intensive and laborious task. With the expansion of new technologies, large video archive can be created and annotated exploiting and storing the expert's knowledge. This paper presents a prototypical study of automatic generation of production rules, in the Horn-clause, using the First Order Inductive Learner (FOIL) algorithm applied to annotated surgical videos of Thoracentesis procedure and of its feasibility to use in context-aware system framework. The algorithm was able to learn 18 rules for surgical workflow model with 0.88 precision, and $0.94 \mathrm{~F} 1$ score on the standard video annotation data, representing entities of the surgical workflow, which was used to retrieve contextual information on Thoracentesis workflow for its application to surgical training.
\end{abstract}

Keywords- surgical workflow; first order inductive learner; ontology; inductive reasoning; context-aware system; data mining

\section{INTRODUCTION}

Surgical workflow modeling is progressively becoming a critical aspect for the efficient management of surgery and in particular for robotic surgeries, to enforce context-awareness and for automating the procedures, eventually. Surgical workflows, are expressing the logical precedence among the surgical activities that build phases of the surgery, which are the intermediate sub-goals which need to be achieved to accomplish the procedure. The standard method for workflow modeling is based on the results of the interviews with domain experts and the modeler's experience [1]. Ontologies are currently under extensive research for surgical workflow modeling, where they aim at providing the procedural knowledge for evaluating the surgical skills or planning the surgery [2] and in some advanced application such as contextawareness [3], robotics [4] and so on. An ontology consists of the domain concepts, properties, and relations, generally "part-of" and "is-a" relationship, and provides a common set of concepts that could help to solve the heterogeneity and complexity of surgical information. However, most of the previous approaches lack the automatic construction and learning aspects of the surgical workflow model that could be used with all the procedures, where the model uses the explicit knowledge formalism representing a common set of concepts. Surgical workflow model could be created with the help of a set of production rules. The manual construction of production rules is a laborious and time-intensive task, often associated with human errors [5]. We believe that a fixed set of production rules with a consistent ontological formalism has potential for several applications in the surgical domain. The observer-based methods rely on the acquisition of surgical data and information [6], such as offline annotation of the surgical videos, where the surgeon records the surgical information by observing the videos, and sensor-based methods [7], such as data obtained through the tracking systems. With the inclusion of robotic surgery, it is very easy to increase the video archives, which can be used for easily retrieving surgical information, once the expert surgeons annotate it. Inductive learning are the methods that enable the production of the general description of information creating knowledge from the specific information provided by a set of examples. During the data and information acquisition in surgery, expert knowledge is supplied by the experienced surgeons through the observer-based methods, e.g. annotating the surgical videos, which constitutes a set of examples, which produces, by induction, the new knowledge or construct a set of constraints inference rules that participate to build the workflow of the surgery. The benefit of modeling the surgical workflow with production rules and the ontological knowledge-base is that the rules are generally more interpretable by humans and they easily allow for possible human correction, amendments, and improvements to the learned knowledge if required. Moreover, video-based extraction of surgical workflow model could capture the variability in surgical workflow.

In literature, different approaches to the surgical workflow modeling can be found [8]. Katić et al. [3] developed an ontology for laparoscopic surgeries and implemented a situation interpretation algorithm, which uses the SQWRL (Semantic Query-enhanced Web Rule Language) [3] rules. However, in this work the queries were constructed manually, with the related problems. Franke et al. [9] developed a rulebased medical device adaptation for the digital operating room workflow management. Franke et al. used RuleML [10] to ground the information entities in the process context and found that two predicates are the efficient expression of timedependent entities. However, RuleML does not have a mechanism to specify the semantic structure on which to evaluate the language elements. Moreover, RuleML is also harder to integrate with the ontology than SWRL. Bertini et al. [11] used FOIL to learn the SWRL rules that were used in the ontology for semantic video event annotation. Blum et al. [12] have modelled and analyzed signals from Laparoscopic videos to segment the surgical workflow using Canonical Correlation Analysis (CCA) and Dynamic Time Warping (DTW), however the approach was not sufficient when the videos missing the whole time-series. Lalys et al. [13] used 
Support Vector Machine (SVM) and Hidden Markov Model (HMM) for surgical phase detection from microscopic videos, however the system was not used to derive surgical workflow model at a lower granularity level e.g. detection of actions. There are different methods to model the surgical workflow and to set the workflow constraints, such as ontological formalism, signal processing, statistical and machine learning [3] [14] [15] [16]. At the best of our knowledge, FOIL and its application for context awareness has not been applied to build a surgical workflow model yet. We implemented an ontology for Thoracentesis in Web Ontology Language (OWL) [17] and production rules for surgical workflow model by performing the reasoning through Semantic Web Rule Language (SWRL) [18]. The main objective of this paper is the application of inductive learning programming, especially First Order Inductive Learner (FOIL) algorithm [19], for automating the generation of first order logic rules, in the form of the Horn-clause, representing the surgical workflow model. Expert knowledge, which is encoded in the observational annotated videos, is used in a knowledge-driven context aware framework, which aims to provide contextual awareness during the surgery.

\section{METHODS}

\section{A. Ontology for Thoracentesis}

Thoracentesis is a procedure that is used to withdraw the fluid from the chest cavity in diseases like pleural effusions [20]. Ontology encompasses surgical entities, as example surgical phases, surgical workflow model, and information about different spatiotemporal activities that occur during Thoracentesis procedure. Ontology for Thoracentesis, as shown in Fig. 1, was built using a top-down approach, where most general concepts of the domain, such as phases (e.g. "Penetration") were analyzed first and thereafter specialized concepts, such as instruments (e.g. "50 ml syringe"), were implemented.

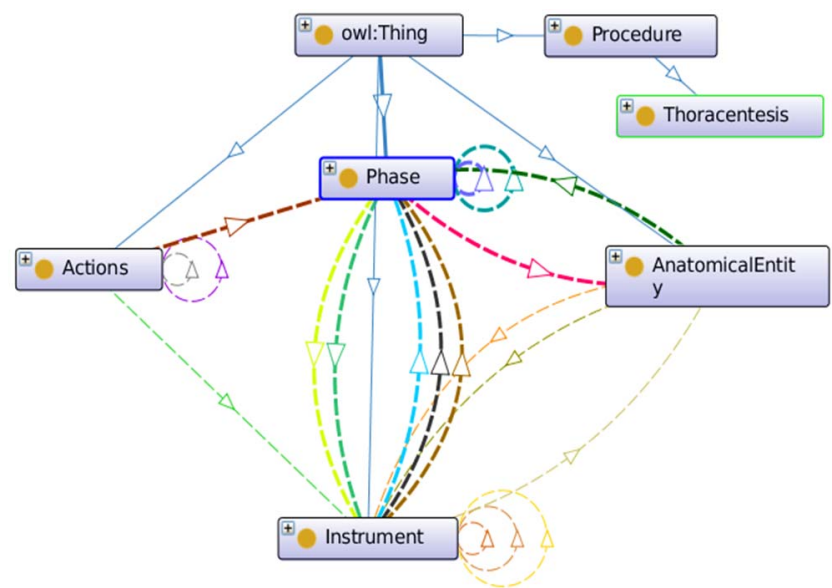

Figure 1. An ontology for Thoracentesis. The rectangles represent classes of the ontology, while blue edges represent hierarchies and other colored dashed line edges represent the relationship, as mentioned in Section II. A, between the classes.
The needed information about Thoracentesis was obtained from a journal article [20], several online web resources, which were verified through HONCode [21] for health information authenticity and analyzed using the methodology described in [22], and an opinion from a physician. The developed ontology represents the optimal substructure. The relations between procedural instances are used in the context interpretation, where the entities representing high-level granularity e.g. phases could be recognized by using the entities that represent low-level granularity e.g. actions and instruments. Greedy algorithms have proven to be useful in solving optimal substructure problem [23] if it can be proved that the solution is optimal at each iterative step. The ontological surgical workflow is specified using the truth conditional semantics, where semantics are specified with the triplets. Each triplet comprises two surgical procedural instances and an instance relationship, which was used to create a new relation for surgical workflow modeling, where each goal relation is also specified using the triplets. Most of the learned ontological relations are transitive, for example, if the action is executed by the instrument and instrument is being using in the phase then the action corresponds to the phase where instrument is being used, to create the surgical workflow model.

A surgical workflow generally consists of the sequence of all the activities performed during the Thoracentesis procedure and their connection to the surgical phases. Our current approach represents individual occurrences during the intra-operative procedure. The phases represented are connected to the instrument through "isUsedInPhase", inverse "hasInstrument" relation and to the body structures through "involvesAnatomicalPart" relation. Moreover, each activity is associated with the phases through "isInPhase" relation. Each instrument is associated with anatomical location through "hasAnatomicalLocation" relation. Each surgical action is associated with the instrument and phase through "hasActionInstrument" and "hasActionInPhase" relations. To form a surgical plan, which are the possible sets of surgical activities divided into phases, different classes and their instances are related to each other by the object properties which help to identify temporal order of the phases, instruments and actions, which are specified in chronological orders e.g. "hasNextInstrument", "hasPreviousInstrument" for instruments and "hasNextPhase", "hasPreviousPhase", "hasPreviousAction" and "hasNextAction" for phases and surgical actions.

\section{B. Video annotations}

We used Anvil annotation research tool [24] to annotate surgical workflow entities, e.g. "Phase", "Anatomical location", "Instrument", and "Action" in the pre-recorded surgical videos (Fig. 2). Four videos for Thoracentesis procedure were collected from the online resources [20] [25] [26] [27], which represents as much as variability in Thoracentesis workflow, after the verification from a midlevel expert clinician. The annotations were done by two midlevel expert clinicians. During the video annotation process, the phases are considered as major objectives to accomplish 
the procedure as per standard procedure workflow. The aim of each phase is to reach/target the principle surgical site. The phase encompasses major changes in the anatomical locations and surgical actions. The instruments and anatomical locations are annotated based on their usage during a phase of the surgery and their correspondence to the appearance in the videos. Anvil allows doing multi-layer annotations on multiple video tracks, where each track specifies different surgical workflow entities, in synchrony. The annotations refer to the activities observed in the videos. Example of such activities are the surgical workflow model entities, that were annotated for a specific period, e.g. from $t_{1}$ (start time) to $t_{2}$ (end time). We did not explicitly specify the dependency between annotations in different tracks. Videos are annotated frame-by-frame representing each entity as individual instance, as controlled vocabularies, e.g. "Penetration" for the ontological class "Phase". So, each of the frame is annotated with the information of surgical workflow entities. Each of these annotations were saved as a text file containing comma separated values of frame number of the video, start time, end time, attribute, and values for an individual surgical workflow model entities.

\section{Rule learning}

We implemented First Order Inductive Learner (FOIL) to learn first order logic rules in the implication form, e.g. " $b$ :$a$, c", constituting the Horn-clause. Ross Quinlan proposed FOIL, which is a greedy algorithm that learns rules to distinguish positive examples from the negative ones. FOIL repeatedly searches for the current best rule and removes all examples which does not satisfy the rule until all the positive examples in the dataset are covered. All expressions are composed of constants (e.g. "Instrument", "BetadineSwab"), variables ("X_", "Y_") and predicates as a set of tuples (e.g. "hasInstrument (Phase, Instrument)", "isUsedInPhase (Instrument, Phase)". Predicates are either true or false. Annotations were used to specify target predicates, e.g. relation between phase and anatomical location, for surgical workflow description. An ontological instance is any constant, or any variable applied to any predicate. A literal is any predicate applied to an ontological instance or a term. A clause is a disjunction of the literals, where all the variables of predicates are universally quantified. In our implementation, we used only the positive literals, e.g. "hasInstrument (SkinOfTheChest, BetadineSwab)". A Hornclause is a clause containing at least one positive literal, as shown in the following:

$$
P \vee \neg Q_{1} \vee \neg Q_{2} \ldots \vee \neg Q_{\mathrm{n}}
$$

where $P$ is the positive literal and $\neg Q_{1}, \neg Q_{2}, \ldots, \neg Q_{\mathrm{n}}$ are the negative literals. It is also equivalent to the following:

$$
\text { IF }\left(Q_{1} \wedge Q_{2} \ldots \wedge Q_{\mathrm{n}}\right) \text { THEN } P
$$

The Horn clause pre-condition, $Q_{1} \wedge Q_{2} \ldots \wedge Q_{\mathrm{n}}$, is called the clause body; and literal $P$ that forms the post-condition is called the clause head.
Each Horn-clause defines some relations given a set of examples belonging to the relations and a set of examples not belonging to the relations. The idea is to create the instances and relations in the ontological assertion box, which contains the assertions on individual instances and correspond to the activities in real-time surgeries. Rules can be encoded as well. We implemented an algorithm, which attempts to satisfy a given set of goals, i.e. literals. If one or more of the goals contains unbound variables, e.g. ontological instances, the algorithm will find every binding for every variable so that the goals are satisfied for searching the literals. The solutions are given as a sequence of ontological instance mappings that satisfy the goals. If there is no mapping that satisfy the goals, then the algorithm yields no results. For example, for an unknown object property relation "phase has instrument", we have positive examples such as "phase has anatomical location" and "anatomical location has instrument" and negative examples "phase does not have anatomical location" and "anatomical location does not have instrument". The FOIL should be able to generate the rule containing the sequence of the variables, e.g. ontological instances, in the form of "phase has instrument" automatically.

We extracted tuples from the video annotations in the form of literals $Q_{1}$ and $Q_{2}$ which forms the rule's precondition and the rule head has been specified with the hypothesis candidates' tuples $P$. The FOIL then tries to find the best literal $Q_{n+1}$, which excludes all negative examples, but includes at least one positive example. This would reduce excessive branching of the rules and also reduce the complexity of individual rule.

The purpose of the clause body is to characterize the information from the tuples representing as much as positive information about the surgical workflow. As mentioned in [19], if current example set contains $P_{i}^{+}$positive tuples and $N_{i}^{-}$negative tuples then information, $I_{i}$, required for rules representing surgical workflow from annotations, is calculated as shown in "eq. (1)".

$$
I_{i}=-\log _{2}\left(\frac{P_{i}^{+}}{P_{i}^{+}+N_{i}^{-}}\right)
$$

Similarly, we calculated new set $P_{i+1}$ for a particular literal $L_{i}$, which is a surgical workflow relation e.g. "instrument has action" as a new information, $I_{i+1}$, similarly as shown in "Eq. (1)". The gain ratio, $G$, compares the performance of a rule before and after adding a new literal e.g. $L_{i}$ and was calculated as shown in "eq. (2)"

$$
G=P_{i}^{++} \times\left(I_{i}-I_{i+1}\right)
$$

where $P_{i}^{++}$of the positive tuples in $P_{i}^{+}$and $N_{i}^{-}$are represented by one or more tuples in $P_{i+1}^{+}$and $N_{i+1}^{-}$. We set a minimum value for the literal gain, e.g. 0.90 , which has to be greater than the gain ratio before determining new literals to be added to the rules. 


\section{Overview of the context-aware software framework}

We developed a knowledge-driven context-aware framework [28], which combines the ontology-based surgical knowledge and the data acquired through 3D image acquisition and processing. The framework was used to create context-awareness by recognizing surgical instrument at a specific instance of the surgical procedure. The framework uses SPARQL query language [29] to process the contextual information. The proposed work, as shown in Fig. 3, will be included in the framework to create the rule-based surgical workflow model that could be able to infer the semantic information, such as an instrument in the phase, dynamically during the procedure to guide surgeons and create the contextual awareness, where we will process the images acquired from laparoscopic videos for feature extraction, through image processing, to be included in SWRL rules and combine the rules generated through inductive learning for creating the surgical workflow model intra-operatively.

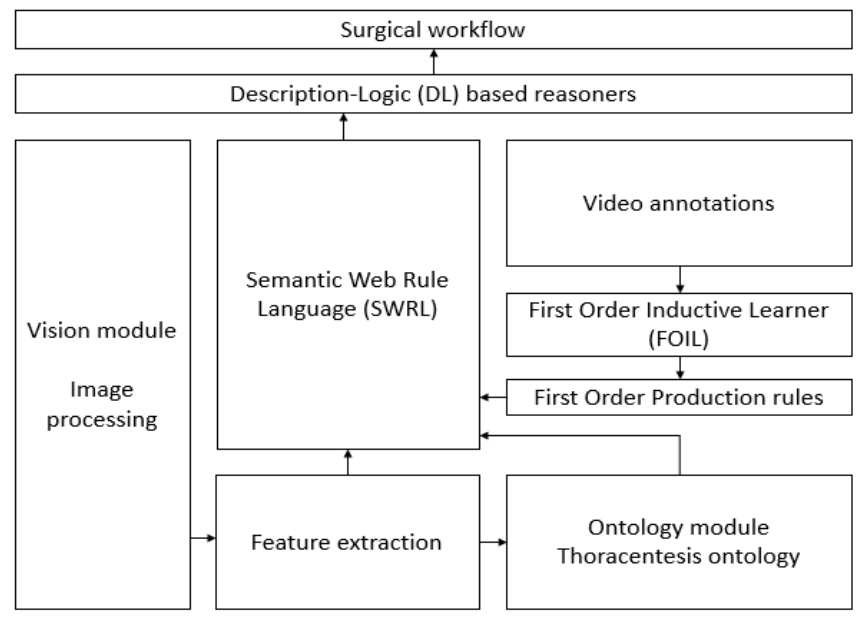

Figure 3. Knowledge-driven context-aware framework; FOIL will be used to generate the SWRL rules.

\section{EXPERIMENTAL PROTOCOL \& RESULTS}

In order to verify FOIL for the generation of the production rules, we implemented three testing protocols.

(1) In first part of the experiments, we applied the proposed method to learn the rules for four different ontological classes "Phase", "Instrument", "Action" and "Anatomical Location". We used positive and negative examples to search literals in the four video annotations data to generate rules. We found out True Positive (TP), False Positive (FP), True Negative (TN), False Negative (FN) to calculate the performance metrics. We obtained the precision, the fraction of the retrieved rules that are relevant to the predicates and recall, the fraction of the predicate instances that are relevant.

(2) To test the effectiveness of the learned first-order production rules in the context-aware framework, we implemented them in the format of SWRL rules to classify the subsumption hierarchies in the ontology and verified the results by inference using the Description-Logic (DL) based reasoners.

(3) Further to that, we measured the relative frequency to test the effectiveness of the learned rules with respect to the manually created rules. The relative frequency $(f)$ of the rules could be calculated as shown in "eq. (3)"

$f=\frac{n_{c}}{n}$

where $n$ is the number of examples the rule matches and $n_{c}$ is the number of examples the rule matches and classifies correctly.

We were able to generate rules with approximately 0.88 precision and $0.94 \mathrm{~F} 1$ score from an annotation representing standard procedure workflow e.g. annotation 4. FOIL could predict more positive rules during the rules extraction from annotation 4, however annotation 4 has very less rules generated and higher precision is non-significant as with annotation 2 , precision approximately $58 \%$, where we were able to generate, as shown in Table 1,13 rules with 8 rules representing the true surgical workflow model, while 5 rules were false positives. Annotation 2 and annotation 4 were equally good annotations for the generation of robust surgical workflow model.

\begin{tabular}{|c|c|}
\hline Video annotations & Number of rules generated \\
\hline Annotation 1 & 10 \\
\hline Annotation 2 & 13 \\
\hline Annotation 3 & 0 \\
\hline Annotation 4 & 9 \\
\hline
\end{tabular}

Table 1. A total number of rule generated for all four annotations

FOIL recall, approximately 1.0, was good, where the algorithm was able to infer the relevant predicate instances or variables, however relational description space was found insufficient for generating the rules. We were not able to obtain any rules and less True Positives with few annotation sets, e.g. annotation 1 and annotation 3, because the sets contained a limited set of literals that could help to yield any results. The precision was very low for annotations 1, approximately 0.44 , and annotations 3 due to the fact that annotations were represented with multiple repetitions of instances, particularly "Instrument", which have been counted as falses, e.g. for annotation dataset 3 . The latter is true because greedy algorithms, e.g. FOIL, could not provide optimal solutions with more than one subproblem satisfying the same goal.

Table 2 represents few rules that were learned with the help of FOIL. A total of 18 rules, from four video annotations, were learned that represent the true logical rules for the surgical workflow model. Considering the fact that 
FOIL generated better rules with annotation 4, we used these rules to test inside our context-aware framework. We found that the rules were able to generate Thoracentsis workflow considering "Phase", "Instrument", "Action" and "AnatomicalLocation" entities. However, the generated workflow was wrong, when the instance of the ontological class contained the relation with more than one instances of the related class. For example, "Anaesthesia" phase has two instruments "Betadine Swab" and "Syringe Filled With Lidocaine". We implemented the first example rule as shown in Table 2, where we found that the next instrument is "Large Syringe" (a $50 \mathrm{ml}$ syringe). However, the classification was wrong because "Large Syringe" was represented as the next instrument for both the instruments, while "Large Syringe" is used to penetrate the pleural cavity after using the "Syringe Filled With Lidocaine" (a $10 \mathrm{ml}$ syringe with anaesthetic liquid) and after anaesthetising the skin of the chest.

\begin{tabular}{|c|}
\hline $\begin{array}{l}\text { Rule for predicate "NextInstrument" (Instrument has next } \\
\text { instrument given the information on instrument } \\
\text { associations) }\end{array}$ \\
\hline $\begin{array}{l}\text { hasNextInstrument (Y0, Y1):- hasInstrument(Y2, Y0), } \\
\text { hasPreviousPhase(Y3, Y2), isUsedInPhase(Y1, Y3) }\end{array}$ \\
\hline Example rule - 1 \\
\hline $\begin{array}{l}\text { Rule for predicate "hasInstrumentInPhase(Phase has } \\
\text { instrument given the information on previous phase) }\end{array}$ \\
\hline $\begin{array}{l}\text { hasInstrumentInPhase }(\mathrm{X} 0, \mathrm{X} 1) \text { :- hasPreviousPhase(Y2, } \\
\mathrm{X} 1) \text {, isUsedInPhase }(\mathrm{X} 0, \mathrm{Y} 2)\end{array}$ \\
\hline Example rule - 2 \\
\hline
\end{tabular}

Rule for predicate "NextInstrument" (Information on next
instrument given the instrument has the anatomical
location)
NextInstrument $(\mathrm{X} 0, \mathrm{X} 1)$ :- hasNextInstrument $(\mathrm{Y} 1, \mathrm{X} 1)$,
hasAnatomicalLocation(Y1, X0)
Example rule - 3

Rule for predicate "hasActionInPhase" (Information on surgical action in a phase given the instrument action and its use in phase)

hasActionInPhase (Y0, Y1):-hasActionInstrument( $\mathrm{Y} 0$,
$\mathrm{Y} 2)$, is UsedInPhase(Y2, Y1 )
Example rule - 4

Table 2. Example rules, obtained using FOIL, where Y0, Y1, Y2, Y3, X0, $\mathrm{X} 1$ represents the ontological instances that satisfy the predicates

We found that the relative frequency of the rules was $78.57 \%$. We considered the generated rules match with the manually created rules when the body of combined literals of differently generated rules also satisfy the clause head. The results show that the algorithm was able to generate the workflow rules that match the manually constructed rules. However, the ordering of the variables and branching of rules were responsible for the wrong classification.

\section{CONCLUSION}

The current prototypical implementation provided only a limited set of production rules to be included in the surgical workflow model. However, the optimization to the annotation process, quality of the videos and robust ontology design would significantly increase the possibility for further potential applications for surgical workflow modeling. In the future work, inductive learning will be integrated into the context-aware framework, by which the system could be able to infer the possible set of production rules that could be useful for automatic generation of the full surgical workflow model and recognizing the surgical instruments for the context awareness, robotic assistance and decision making. We also envisage integrating the feature extraction, e.g. for the instrument recognition, from the videos, and the production rules for the robust surgical workflow management and application to the autonomous robotic surgery.

\section{ACKNOWLEDGMENT}

This project has received funding from the European Union's Horizon 2020 research and innovation programme under grant agreement No. H2020-ICT-2016-732515.

\section{REFERENCES}

[1] T. Neumuth, "Surgical Process Modeling - Thoery, Methods and Applications", Habilitation thesis, Faculty of Medicine, Universiy of Leipzig, Leipzig, Germany, 2012, doi: 10.13140/RG.2.1.1516.4244.

[2] A. Machno, P. Jannin, O. Dameron, W. Korb, G. Scheuermann, J. Meixensberger, "Ontology for assessment studies of human computer interaction in surgery", Artificial Intelligence in Medicine, vol. 63, no. 2,2015 , p. $73-84$.

[3] D. Katić, C. Julliard, A.-L. Wekerle, H. Kenngott, B. P. Müller-Stich, R Dillmann, S. Speidel, P. Jannin, B. Gibaud, "LapOntoSPM: an ontology for laparoscopic surgeries and its application to surgical phase recognition", International Journal of Computer Assisted Radiology and Surgery, 2015, 10(9), pp. 1427-34.

[4] H. Fujita, I.J. Rudas, J. Fador, M. Kurematsu, J. Hakura, "Fuzzy reasoning for medical diagnosis-based aggregation on different ontologies", in proceedings of 7th IEEE International Symposium on Applied Computational Intelligence and Informatics (SACI), 2012, pp. 137-146.

[5] D. Georgiana and W. Rui, "Inference rules and their application to recognizing textual entailment", Proceedings of the 12th Conference of the European Chapter of the Association for Computational Linguistics, March 30-April 03, 2009, p. 211-219, Athens, Greece

[6] T. Neumuth, P. Jannin, G. Strauß, J. Meixensberger, O. Burgert, "Validation of knowledge acquisition for surgical process models", Journal of the American Medical Informatics Association. 2009; 16(1): pp. $72-80$.

[7] T. Neumuth, C. Meißner, "The impact of information fusion strategies for online recognition of activities for surgical process modeling", International Journal of Computer Assisted Radiology and Surgery. 2012; 7(2), pp. 297-304.

[8] L. Florent and P. Jannin, "Surgical process modelling: a review," International Journal of Computer Assisted Surgery and Radiology, Vol. 9, Issue 3, 2014, pp. $495-511$.

[9] S. Franke and T. Neumuth, "Rule-based medical device adaptation for the digital operating room", in proceedings of IEEE conference of Engineering in Medicine and Biology Society, 2015, pp. 1733-1736.

[10] B. Harold, "The RuleML Family of web rule languages", in PPSWR'06 proceedings of the 4th international conference on Principles and practice of Semantic Web Reasoning, 2006, pp. 1-17. 
[11] M. Bertini, A. Del Bimbo, G. Serra, "Learning Rules for Semantic Video Event Annotation," In: M. Sebillo, G. Vitiello, G. Schaefer (eds) Visual Information Systems. Web-Based Visual Information Search and Management. VISUAL 2008. Lecture Notes in Computer Science, vol 5188, 2008, Springer, Berlin, Heidelberg.

[12] T. Blum, H. Feußner, N. Navab, "Modeling and segmentation of surgical workflow from laparoscopic videos", in proceedings of MICCAI 2010 International conference on Medical Image Computing and Computer-Assisted Interventions, Lecture notes in Computer Science, 2010, Vol. 6363, pp. 400-407, Springer, Berlin, Heidelberg.

[13] F. Lays, L. Riffaud, X. Morandi, P. Jannin, "Surgical phases detection from microscopic videos by combining SVM and HMM, in: B. Menze, G. Langs, Z. Tu, A. Criminisi, Medical Computer Vision. Recognition Techniques and Applications in Medical Imaging, Lecture notes in Computer Science, 2010, Vol. 6533, pp. 54-62, Springer, Berlin.

[14] O. Dergachyova, D. Bouget, A. Huaulmé, X. Morandi, P. Jannin, "Automatic data-driven real-time segmentation and recognition of surgical workflow", International Journal of Computer Assisted Surgery and Radiology, 2016, 11(6), pp. 1081-90.

[15] F. Nessi, E. Beretta, C. Gatti, G. Ferrigno and E. De Momi, "Gestemefree context-aware adaptation of robot behaviour in human-robot cooperation”, Artificial Intelligence in Medicine, 2016, 74, pp. 32-43.

[16] Y. Kassahun, R. Perrone, E. Do Momi, E. Berghöfer, L. Tassi, M.P. Canevini, R. Spreafico, G. Ferringo and F. Kirchner, "Automatic classification of epilepsy types using ontology-based and geneticbased machine learning", Artificial Intelligence in Medicine, 2014, 61(2), pp. 79-88.

[17] G. Antoniou and F. van Harmelen, "Web Ontology Language: OWL", In: Handbook on Ontologies in Information Systems, Germany, Springer, 2003, pp. 67-92

[18] I. Horrocks, P. Patel-Scheider, H. Boley, S. Tabet, B. Grosof, M. Dean (accessed 2017 Jan 20) "SWRL: A Semantic Web Rule Langauge combining OWL and RuleML". W3C Member Submission 2004, Retrieved from https://www.w3.org/Submission/SWRL.
[19] J.R. Quinlan, "Learning Logical Definitions from Relations" Machine Learning, 1990, Vol. 5, Issue 3, pp. 239-266.

[20] T. W. Thomsen, J. DeLaPena, G. S. Setnik, "Thoracentesis Videos in clinical medicine", The New England Journal of Medicine, 355(15), (2006), e16.

[21] Health On the Net Code of Conduct [Internet] Health On Net Foundation, Switzerland. (2015 Nov) [accessed 2016 Jan 17]. Retrieved from: https://www.healthonnet.orhg/HONcode

[22] T. Neumuth, G. Strauß, J. Meixensberger, H. U. Lemke, O. Burgert, "Acquisition of process descriptions from surgical interventions" In DEXA 2006 LNCS, vol. 4080, Springer, S. Bressan, J. Kung, R. Wagner (Heidelberg, Germany), pp. 602-611.

[23] T.H. Corman, C.E. Leiserson, R.L. Rivest, C. Stein, "Introduction to algorithms, third edition", USA, MIT Press, 2009.

[24] M. Kipp, "Spatiotemporal Coding in ANVIL," Proc. of the 6th international conference on Language Resources and Evaluation (LREC-08), pp. 2042-2045, 5/2008, Marrakech, Morocco.

[25] PACES MRCP UK resources (2009, Jun 7), "Thoracentesis" [video file], Retrieved from https://www.youtube.com/watch?v=2ZRip1STS $\mathrm{SQ} \& \mathrm{t}=74 \mathrm{~s}$.

[26] InfoTel-Med (2009, Mar 7), "Toracocentesis" [video file], Retrieved from https://www.youtube.com/watch? $\mathrm{v}=\mathrm{xgmP4F} 6 \mathrm{aR} 3 \mathrm{Q}$.

[27] TrinsicAnimation (2013, Jan 31), "Thoracentesis Medical Animation" [Video file], Retrieved from https://www.youtube.com/watch?v=z0 $\mathrm{dCL} 4 \mathrm{CHGSk} \& \mathrm{t}=4 \mathrm{~s}$.

[28] H. Nakawala, G. Ferrigno, E. De Momi, "Toward a knowledge-driven context-aware system for surgical assitance", Journal of Medical Robotics Research, 2017, vol.2, no. 3, 1740007 (Online ready).

[29] E. Prud'hommeaux and A. Seaborne "SPARQL query lanuaguge for RDF. W3C recommendation. (2008) [accessed 2017 Feb 18]. Available from: https://www.w3.org/TR/rdf-sparql-query/

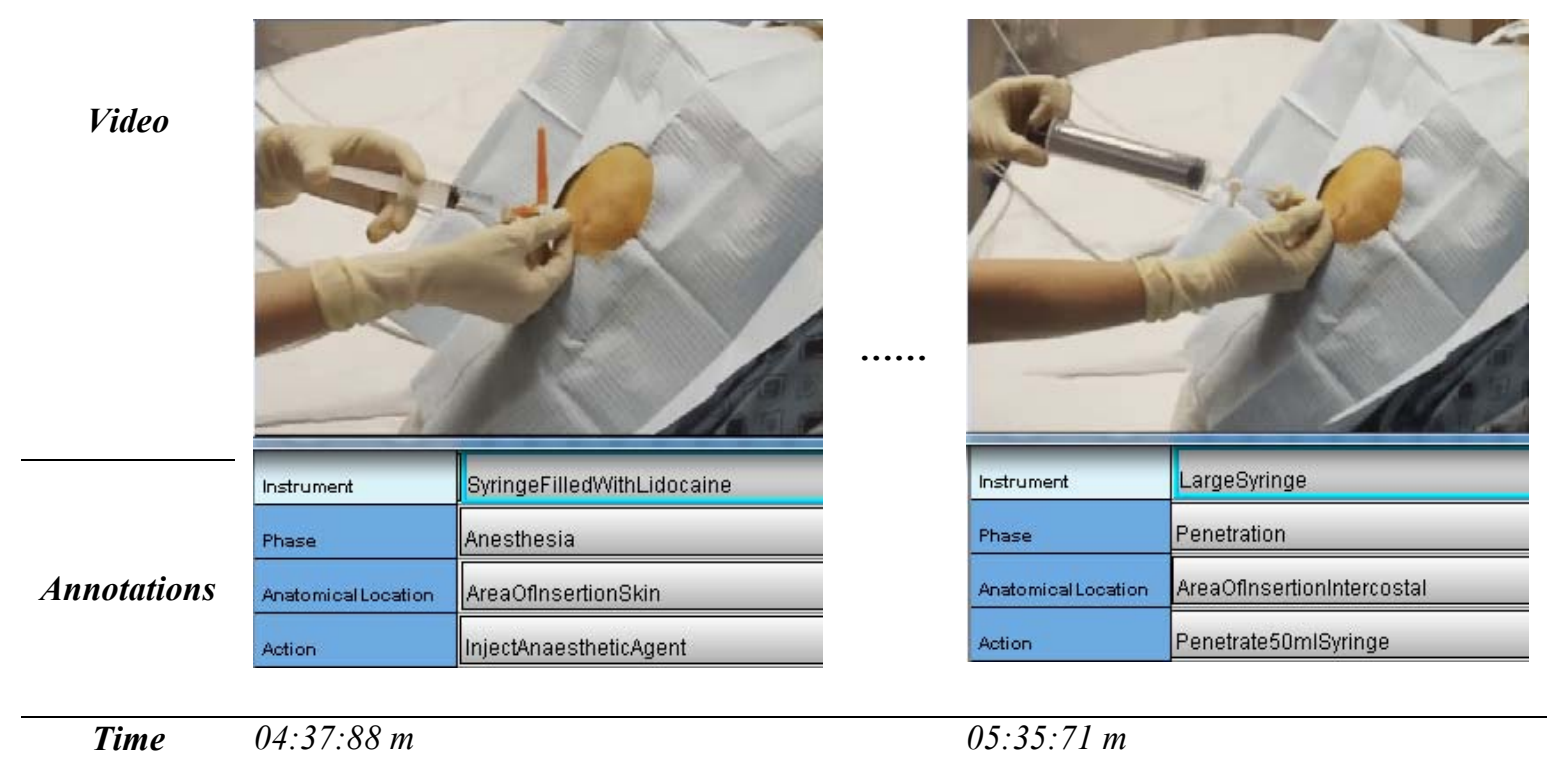

Figure 2. Example of the annotation of the surgical video, where "SyringeFilledWithLidocaine" and "LargeSyringe" represents instruments, $10 \mathrm{ml}$ syringe with filled-in lidocaine/xylocaine liquid and $50 \mathrm{ml}$ syringe respectively. "Anaethesia" and "Penetration" represents the phases of the surgery. "AreaOfInsertionSkin" and "AreaofInsertionIntercostal" represents the marked over area of the skin of chest, based on physical assessment, and 1 or $2 \mathrm{~cm}$ intercostal spaces below the level of effusion as anatomical locations respectively. "InjectAnaestheticAgent" and "Penetrate50mlSyringe" represents the surgical actions required to complete the surgical activity. 